\title{
Estilos de crianza y su relación con el comportamiento prosocial en adolescentes
}

\section{Parenting styles and their relationship with prosocial behavior in adolescents}

1 Nicole Bárbara Pérez Paredes https://orcid.org/0000-0001-8564-9252 Universidad Técnica de Ambato, Carrera de Psicología Clínica, Ambato-Ecuador, nperez7775@uta.edu.ec

2 Mayra Lucía Rodríguez Pérez https://orcid.org/0000-0003-0383-998X Universidad Técnica de Ambato, Carrera de Psicología Clínica, Ambato-Ecuador, ml.rodriguez@uta.edu.ec con el comportamiento prosocial en adolescentes. ConcienciaDigital, 6(1.4), 593-605. https://doi.org/10.33262/concienciadigital.v6i1.4.2016

CONCIENCIA DIGITAL, es una Revista Multidisciplinar, Trimestral, que se publicará en soporte electrónico tiene como misión contribuir a la formación de profesionales competentes con visión humanística y crítica que sean capaces de exponer sus resultados investigativos y científicos en la misma medida que se promueva mediante su intervención cambios positivos en la sociedad. https://concienciadigital.org .

La revista es editada por la Editorial Ciencia Digital (Editorial de prestigio registrada en la Cámara Ecuatoriana de Libro con No de Afiliación 663) www.celibro.org.ec 


\section{Palabras} claves:

crianza, conducta social, empatía y adolescentes.

\section{Keywords:}

parenting, social behavior, empathy and adolescents.

\section{Resumen}

Introducción. La manera en que los padres crían y educan a sus hijos o los estilos de crianza que emplean son determinantes para varias esferas de la vida, entre ello se encuentra la conducta prosocial que son las acciones que se realizan en beneficio de otras personas sin esperar retribución de ningún tipo. Objetivo. Identificar la relación de los estilos de crianza y el comportamiento prosocial en adolescentes. Metodología. La muestra estuvo conformada por 96 estudiantes pertenecientes a primero, segundo y tercero de bachillerato general unificado, de la provincia de TungurahuaEcuador, durante el año electivo 2021-2022, mediante un estudio de enfoque cuantitativo, de alcance descriptivo-correlacional y de corte transversal. Se utilizó el Cuestionario de Dimensión y Estilos de Crianza y la Escala original de Prosocialidad de Caprara. Resultados. Se determinó que el estilo de crianza que prevalece entre los adolescentes es el autoritativo, seguido del permisivo. Con respecto al comportamiento prosocial se encontró que predomina la conducta prosocial y posteriormente la empatía y apoyo emocional. Asimismo, se demostró que las mujeres presentan mayor tendencia prosocial en relación con los hombres, identificando diferencias significativas. Al mismo tiempo, se evidenció una correlación estadísticamente significativa entre estilos de crianza y comportamiento prosocial. Conclusión. Se concluyó que los estilos de crianza empleados por los cuidadores principales están relacionados con el comportamiento prosocial de los adolescentes, de manera que dentro de la familia se internalizan los valores y normas.

\section{Abstract}

Introduction. The way in which parents raise and educate their children or the parenting styles they use are decisive for various spheres of life, including prosocial behavior, which are actions carried out for the benefit of other people without expecting retribution of any kind. Objective. Identify the relationship between parenting styles and prosocial behavior in adolescents. Methodology. The sample consisted of 96 students belonging to the first, second and third of unified general high school, from the province of Tungurahua-Ecuador, during the scholar year 20212022, through a study with a quantitative approach, descriptivecorrelational scope and cutcross. The Parenting Dimension 
and Styles Questionnaire and the original Caprara Prosociality Scale were used. Results. It was determined that the parenting style that prevails among adolescents is authoritative, followed by permissive. With regard to prosocial behavior, it was found that prosocial behavior predominates and later empathy and emotional support. Likewise, it was shown that women present a greater prosocial tendency in relation to men, identifying significant differences. At the same time, a statistically significant correlation was found between parenting styles and prosocial behavior. Conclusion. It was concluded that the parenting styles used by the main caregivers are related to the prosocial behavior of adolescents, so that values and norms are internalized within the family.

\section{Introducción}

La familia es considerada el núcleo de la sociedad, por tal razón desempeña un rol fundamental que es el de la socialización primaria, es decir es la encargada de la formación e internalización de valores en los hijos lo que les permitirá la inclusión a la sociedad (Paez \& Rovella, 2019). En ocasiones cuando existe una inadecuada socialización por parte de los padres o cuidadores principales conlleva a cometer actos en donde prevalece los interés individua les lo que ocasiona un deterioro en la sociedad (Mestre et al., 2004).

En este aspecto, los estilos de crianza son la manera que tienen los padres de responder y reaccionar ante las emociones de un niño, estas formas de reaccionar tienen consecuencias que afectan en el estadio infantil y son sumamente importantes en la vida de todo ser humano, debido a que mientras el individuo crece se va produciendo la educación y socialización a través de la adquisición de nuevas habilidades (Villavicencio et al., 2020). Además, los estilos de crianza forman parte de las relaciones familiares, en donde se puede evidenciar el rol que cumplen los padres en la educación y formación de los hijos, es así que los padres generalmente tienen una idea predispuesta y aprendida de sus padres o la sociedad, lo que ocasiona que ciertas actitudes repitan con sus hijos (Ramírez, 2005).

Baumrind determinó tres estilos de crianza que permiten a los padres o cuidadores principales controlar las conductas de sus hijos estos son autoritario, permisivo y autoritativo (Velásquez et al., 2015).

El estilo autoritario se caracteriza en que los padres establecen una dinámica de forma unidireccional, en el cual lo fundamental es la obediencia, es decir los hijos deben hacer lo que los padres digan u ordenen (Bohorquez et al, 2021). Los padres dedican gran parte de 
su tiempo al control y supervisión de las acciones y actitudes de los niños, que deben estar dentro de los parámetros y normas impuestas, se instaura un castigo o recompensa basado en la obediencia que tenga la persona a las instrucciones dictadas, por lo que dentro de este estilo no se medía situaciones con los hijos (Peña et al., 2015).

Mientras que no restrictivo o permisivo este estilo de crianza se caracteriza porque al niño se le otorga total autonomía y no se establece límites en los lugares públicos, la familia u otros escenarios y si se lo hace son muy pocos (Aldana \& Hernández, 2018). Esto provoca aceptación total a las acciones o impulsos, dando lugar a una a una libración de todo medio autoritario, restrictivo o tipo de control (Quezada Fernández, 2015).

Finalmente el estilo autoritario o democrático en este estilo de crianza parental también es primordial las normas de comportamiento y altas expectativas que tienen los padres sobre el niño, pero se escucha las dudas y se atiende las necesidades que tenga el infante, en este modelo se acompaña el desarrollo comportamental del niño (Pinheiro \& Assunção, 2020). Se practica la escucha activa y se guía las decisiones del menor mediante una herramienta importante que son los consejos (Franco et al., 2014). Este modelo genera la construcción de individuos con altos niveles de autoestima, autosuficiencia, y tolerancia esto se puede evidenciar con la habilidad social con la que se desempañan $y$ en las relaciones interpersonales tanto con su familia como con sus pares (Henao et al., 2007).

Mientras que el comportamiento prosocial se define como la tendencia a realizar acciones que buscan un efecto beneficioso sobre los demás y pertenece al ámbito de los hábitos, las prácticas y el modo común de interacción social. Algunos autores mencionan que el verdadero comportamiento prosocial es aquel en donde la ayuda que brinda una persona es aquella que no tiene motivación de ningún tipo, por lo tanto no hay recompensa material o extrínseca (Correa Duque, 2017; Balabanian et al., 2020).

Además, el comportamiento prosocial es una variable ambiental, cognitiva y emociona l, que determina las maneras de razonamiento más internalizadas, las cuales están conectadas positivamente a las conductas prosociales (Mestre et al., 2006). Asimismo, la prosocialidad se ve influenciada por varios factores: culturales, familiares, escolares y personales y todos estos, influyen para que la persona desarrolle su conducta prosocial, tomando en cuenta lo que mencionan algunos autores que el altruismo es muy común y por ende la conducta prosocial es universal (Garaigordobil, 2014).

La prosocialidad comprende las conductas y actitudes encaminadas al bienestar, tanto de individuos como de colectivos, por medio de actos solidarios hacia los demás, basándose en sentimientos, creencias y valores que orientan conductas de ayuda sin ningún tipo de retribución (Rico et al, 2021). Es así que, la prosocialidad está relacionada con los estilos parentales pero también se ve mediada por aspectos emocionales como la empatía y apoyo emocional (Llorca et al., 2017). 
La empatía es la capacidad que tiene una persona para experimentar las respuestas afectivas y comprender las perspectivas de otra persona, esta respuesta emocional se vincula a la conducta prosocial, debido a que aumenta la preocupación empática por los otros, la cual está encaminada a beneficiar a los demás de manera voluntaria (Yoo et al., 2013). En los adolescentes la empatía se ve influenciada por experiencias tempranas, debido a que, el desarrollo emocional se ve afectada por los cuidadores principales y las relaciones interpersonales más cercanas (Valdivia et al., 2020).

También está presente el aspecto de apoyo emocional, que se ve directamente influenciado por los padres, quienes cumplen el rol fundamental de satisfacer las necesidades emociona les de los niños en los primeros años de vida (Popp et al., 2019), por esa razón, el apoyo emocional que se brinde puede depender de la estabilidad y capacidad que tenga cada persona, debido a que, en ocasiones tiende a afectar indirectamente al individuo.

\section{Metodología}

La presente investigación es de enfoque cuantitativo, de alcance descriptivo y correlacional y de corte transversal. Con una población constituida por 97 estudiantes, pertenecientes a primero, segundo y tercero de bachillerato general unificado (BGU) de la Unidad Educativa "Vicente Anda Aguirre" en la ciudad de Mocha, provincia Tungurahua-Ecuador, desarrollada en el año lectivo 2021-2022. La muestra estuvo conformada por 96 estudiantes (48 mujeres y 48 hombres), con edades comprendidas entre los 15 y 18 años, mediante un muestreo no probabilístico, de tipo intencional o deliberado, considerando las siguientes características:

Aceptación y firma del consentimiento informado por parte de los padres y/o tutores legales.

Edad comprendida entre 15 y 18 años.

Adolescentes que no presenten ningún tipo de discapacidad.

Adolescentes que no se encuentren en tratamiento psicológico y/o farmacológico.

En el presente estudio no se utilizaron métodos invasivos que pudieran generar algún riesgo en los participantes. Con respecto a los aspectos éticos, en la investigación se contó con la autorización de los organismos competentes, el Distrito 18D06 y el rector de la Unidad Educativa Mocha-Ecuador. De igual manera, se obtuvo la aceptación de los padres o tutores legales mediante la firma del consentimiento informado, quienes accedieron de la manera voluntaria a la participación de los adolescentes. En el trabajo, se respetó la confidencialidad de los datos procesados, los mismos que se emplearon con fines académicos y científicos.

Los instrumentos fueron aplicados de manera presencial, siendo estos la escala de conducta prosocial de Caprara y el cuestionario de dimensión y estilos de crianza. 
la escala de conducta prosocial (ECP). Este instrumento evalúa la conducta de ayuda y empatía, mediante 16 ítems tipo Likert, 6 ítems que evalúan conducta prosocial como la confianza, conducta de ayuda y simpatía, 4 ítems que evalúan empatía y apoyo emocional y los 6 ítems restantes son de control de aquiescencia. Esta escala presenta índice de fiabilidad de 0.76 en el Alpha de Cronbach (Rodríguez et al., 2017).

Cuestionario de dimensión y estilos de crianza (styles and dimensions questionnaire): el instrumento consta de 62 ítems tipo Likert, los cuales se están organizados en tres dimensiones, que corresponden a los tres tipos de estilos de crianza que son autoritario, autoritativo y permisivo. La evaluación realizada a través del Alfa de Cronbach para cada dimensión dieron como resultado: dimensión autoritativo 0,91 , dimensión autoritaria 0,86 y dimensión permisiva 0,75 (Velásquez et al., 2015).

La aplicación de los test psicológicos se desarrolló de manera colectiva en cada salón de clase de la Unidad Educativa, en donde se explicaron los objetivos del estudio. Para el análisis estadístico de los resultados, se aplicó el programa estadístico SPSS versión 28. En el procesamiento de los datos se utilizó la estadística descriptiva (comparación de medias), también se empleó la estadística diferencial con pruebas no paramétricas, considerando que la población no fue normal, para comparar grupos según el sexo se utilizó la U de Mann- Whitney y la correlación de Spearman para identificar la relación entre las variables estilos de crianza y comportamiento prosocial.

\section{Resultados}

$\mathrm{Al}$ analizar las medias entre las puntuaciones de los tres estilos de crianza, se encontró que el que más prevalente es el Autoritativo $\mathrm{M}=3,55$, seguido del Permisivo $\mathrm{M}=2,36$ y finalmente el Autoritario $\mathrm{M}=2,26$

\section{Tabla 1}

Estilos de crianza

\begin{tabular}{lccc}
\hline \multicolumn{1}{c}{ Estilos de crianza } & Autoritativo & Autoritario & Permisivo \\
\hline Media & 3,55 & 2,26 & 2,36 \\
Mediana & 3,56 & 2,2 & 2,27 \\
Moda & 3 & $2 *$ & 2 \\
Rango & 3 & 2 & 2 \\
Mínimo & 2 & 1 & 2 \\
Máximo & 5 & 4 & 4 \\
\hline
\end{tabular}

Fuente: Pérez y Rodríguez 
Con respecto al comportamiento prosocial, se comparó las medias de sus dos aspectos y se encontró una predominancia de la conducta prosocial $\mathrm{M}=3,65$ seguida de la dimensión

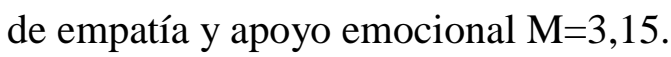

Tabla 2

Aspectos de tendencia prosocial

\begin{tabular}{lcc}
\hline Comportamiento Prosocial & Conducta Prosocial & Empatía y apoyo emocional \\
\hline Media & 3,65 & 3,15 \\
Mediana & 3,67 & 3,25 \\
Moda & 4 & 3 \\
Mínimo & 2 & 1 \\
Máximo & 5 & 5 \\
\hline
\end{tabular}

Fuente: Pérez y Rodríguez

Al comparar los aspectos del comportamiento prosocial con el sexo, se aplicó la prueba U de Mann Whitney y se observó que la conducta prosocial predomina en mujeres RM=55,13 mientras que en hombres $\mathrm{RM}=41,88$, encontrándose una diferencia estadísticamente significativa $(\mathrm{U}=834,000 \mathrm{p}<0,05)$. Con respecto a la empatía y apoyo emocional la puntuación más alta que se obtuvo fue en mujeres $\mathrm{RM}=54,14$ que en hombres $\mathrm{RM}=42,86$, evidenciándose diferencias estadísticamente significativas.

Tabla 3

Aspectos de tendencia prosocial y sexo

\begin{tabular}{|c|c|c|c|c|c|c|}
\hline \multicolumn{7}{|c|}{ Sexo } \\
\hline \multirow[t]{2}{*}{ Aspectos } & \multicolumn{2}{|c|}{ Hombres } & \multicolumn{2}{|c|}{ Mujeres } & \multicolumn{2}{|c|}{ Total } \\
\hline & No & $\%$ & ni & $\%$ & no & $\%$ \\
\hline Conducta Prosocial & 48 & 41,88 & 48 & 55,13 & 96 & 100 \\
\hline U de Mann-Whitney & \multicolumn{6}{|c|}{834} \\
\hline Sig. asintótica (bilateral) & \multicolumn{6}{|c|}{0,019} \\
\hline Empatía y apoyo emocional & 48 & 42,86 & 48 & 54,14 & 96 & 100 \\
\hline U de Mann-Whitney & \multicolumn{6}{|c|}{881,5} \\
\hline Sig. asintótica (bilateral) & \multicolumn{6}{|c|}{0,047} \\
\hline
\end{tabular}

Fuente: Pérez y Rodríguez

En relación con los valores de Rho de Spearman se pudo establecer la relación estadísticamente significativa entre conducta prosocial y el estilo autoritativo ( $\mathrm{p}=0,001)$, la cual fue positiva baja $(\mathrm{Rho}=0,323)$; de la misma manera se observó una relación significativa entre 
la conducta prosocial y el estilo permisivo $(\mathrm{p}=0,032)$ siendo esta positiva baja $(\mathrm{Rho}=0,218)$. De la misma manera se obtuvo una relación estadísticamente significativa entre empatía y apoyo emocional con el estilo autoritativo $(\mathrm{p}=0,001)$ la cual fue igual positiva baja ( $\mathrm{Rho}=0,337)$.

Tabla 4

Correlación estilos de crianza y comportamiento prosocial

\begin{tabular}{lccc}
\hline \multicolumn{1}{c}{ Conducta prosocial } & Autoritativo & Autoritario & Permisivo \\
\hline Rho de Spearman & $0,323^{* *}$ & 0,05 & $0,218^{*}$ \\
Sig. (bilateral) & 0,001 & 0,628 & 0,032 \\
\hline Empatía y apoyo emocional & Autoritativo & Autoritario & Permisivo \\
\hline Rho de Spearman & $0,337^{* *}$ & $-0,074$ & 0,143 \\
Sig. (bilateral) & $<0,001$ & 0,476 & 0,164 \\
\hline
\end{tabular}

Fuente: Pérez y Rodríguez

\section{Discusión}

A partir de los estilos de crianza que emplean los padres con los adolescentes, la forma de educar y afianzar los valores se puede manifestar que son fundamentales para la manera de comportarse y actuar de manera prosocial con los demás.

En referencia con los resultados de estilos de crianza se observa el predominio del estilo autoritativo con 3,55, lo que se asemeja con la investigación realizada en Colombia en una población con diferentes estratos económicos (Aguirre Dávila, 2015), de igual manera se observa el trabajo realizado en un grupo de adolescentes españoles con resultados similares (Mestre et al., 2007). A diferencia del estudio realizado en Perú en el cual menciona que gran parte de los padres mantienen un estilo de crianza autoritario (Castillo Castro, 2016).

El aspecto del comportamiento prosocial con mayor prevalencia fue la conducta prosocial con 3,65 similar a los resultados encontrados en adolescentes escolarizados con discapacidad intelectual leve en donde se evidencian altos niveles de prosocialidad en los sujetos estudiados, seguido de la empatía y apoyo emocional (Montiel y González, 2021). Además se encontró un estudio desarrollado en 359 adolescentes argentinos de clase media, el cual corrobora con los resultados obtenidos sobre la conducta prosocial alta (Balabanian et al., 2020), sin embargo en un estudio realizado en Colombia con un grupo de adolescentes desvinculados de grupos armados, menciona

que los aspectos de empatía y apoyo emocional presentaron mayor incidencia que la conducta prosocial (Gómez Tabares, 2019).

Al comparar el comportamiento prosocial y el sexo se observa el predominio de conducta 
prosocial en mujeres con 55,13, así como también la empatía y apoyo emocional con 54,14 , encontrándose diferencias significativas entre los grupos, lo que se pudo corroborar con los estudios desarrollados en adolescentes españoles (Mestre et al., 2007) y en un grupo de 518

adolescentes argentinos pertenecientes a establecimientos públicos, en los cuales también se obtuvieron los resultados similares a esta investigación (Paez \& Rovella, 2019).

Se estableció una relación positiva y estadísticamente significativa entre los estilos de crianza y comportamiento prosocial al igual que la investigación realizada en 233 adolescentes de establecimientos de secundaria publica en Estados Unidos, en este estudio se manifiesta que las prácticas de los padres se asocian directamente con el comportamiento prosocial de los adolescentes (Carlo et al., 2007), además se evidencia la investigación desarrollada en Estados Unidos entre padres e hijos, que coincide con los resultados obtenidos en este estudio (Yoo et al., 2013). Lo que difiere del trabajo desarrollado en 1285 adolescentes en el cual se obtuvo como resultado que los estilos parentales están en segundo lugar en el perfil con sujetos con baja disposición prosocial (Mestre et al., 2004).

\section{Conclusiones}

- Se determinó que el estilo de crianza que prevalece en los adolescentes es el estilo autoritativo o democrático en el cual se refleja que gran parte de los padres de familia atienden y escuchan las necesidades de sus hijos.

- En los aspectos de tendencia prosocial, el que se identifica mayormente es el de la conducta prosocial que corresponde a las actitudes que tienen los adolescentes con los demás, proporcionando su ayuda sin esperar recompensas de ningún tipo.

- Con respecto a los aspectos de tendencia prosocial, las puntuaciones más relevantes se encuentran en las mujeres, siendo la conducta prosocial más prevalente seguida de la empatía y apoyo emocional, lo que también se pudo observar en hombres con puntuaciones más bajas, mostrando diferencias significativas.

- Se pudo determinar que los estilos de crianza empleados por los padres como el autoritativo y permisivo están estrechamente relacionados con el comportamiento prosocial que presentan los adolescentes, debido a que los valores y educación impartida por parte de los padres es fundamental para dicho comportamiento.

- Dentro de este estudio se evidencia que la información sobre el tema es escasa, por lo que se propone seguir realizando investigaciones sobre este tema, para generar nuevos conocimientos, además es necesario continuar trabajando en la población adolescente, por ser un grupo etario, que por su edad necesita de orientación y guía para un adecuado desarrollo a nivel integral. 


\section{Referencias bibliográficas}

Aguirre Dávila, E. (2015). Prácticas de crianza, temperamento y comportamiento prosocial de estudiantes de educación básica. Revista Latinoamericana de Ciencias Sociales, Niñez y Juventud, 13(1). https://doi.org/10.11600/1692715x.13113100314

Aldana Camacho, L. \& Hernández Rincón, S. (2018). Reconocimiento emocional: Entendiendo los estilos de crianza. (Tesis). Corporación Universitaria Minuto de Dios]. https://repository.uniminuto.edu/handle/10656/6793

Balabanian, C. \& Lemos, V. (2020). El rol de la atribución en el comportamiento prosocial adolescente. Revista Interdisciplinaria, 37(2), 129-142. https://doi.org/10.16888/interd.2020.37.2.8

Bohorquez Jaramillo, J., Cevallos Ramón, R. \& Belduma Cabrera, K. (2021). Estilos de crianza y problemas conductuales en caso con discapacidad intelectual leve. [Tesis de Maestría, Universidad Técnica de Machala] 44. http://repositorio.utmachala.edu.ec/bitstream/48000/17158/1/proyecto\%20estu dio\%20de\%20caso $\% 202021 \% 20$ \%20Julisa\%20Lizbeth\%20Bohorquez\%20Jaramillo.pdf

Carlo, G., McGinley, M., Hayes, R., Batenhorst, C. \& Wilkinson, J. (2007). Parenting Styles or Practices? Parenting, Sympathy, and Prosocial Behaviors Among Adolescents. The Journal of Genetic Psychology, 168(2), 147-176. https://doi.org/10.3200/GNTP.168.2.147-176

Castillo Castro, P. (2016). Estilos de crianza de los padres de familia de los alumnos del 2 do grado de educación primaria de la I.E. María Goretti de Castilla [Tesis de Pregrado, Universidad de Piura]. https://pirhua.udep.edu.pe/handle/11042/2564

Correa Duque, M. (2017). Aproximaciones epistemológicas y conceptuales de la conducta prosocial. Zona Próxima, 27, 3-21. https://doi.org/10.14482/zp.27.10978

Franco Nerín, N., Pérez Nieto, M. \& De Dios Pérez, M. (2014). Relación entre los estilos de crianza parental y el desarrollo de ansiedad y conductas disruptivas en niños de 3 a 6 años. Revista de Psicología Clínica con Niños y Adolescentes. https://www.redalyc.org/articulo.oa?id=477147184006

Garaigordobil, M. (2014). Conducta prosocial: El papel de la cultura, la familia, la 
escuela y la personalidad. Revista Mexicana de Investigación en Psicología. https://www.medigraphic.com/pdfs/revmexinvpsi/mip-2014/mip142d.pdf

Gómez Tabares, A. S. (2019). Conductas prosociales y su relación con la empatía y la autoeficacia para la regulación emocional en adolescentes desvinculados de grupos armados ilegales. Revista Criminalidad, 61(3), 221-246.

Henao López, G., Ramírez Palacio, C. \& Ramírez Nieto, L. (2007). Las prácticas educativas familiares como facilitadoras del proceso de desarrollo en el niño y niña. El Ágora USB, 7(2), 233-240.

https://www.redalyc.org/articulo.oa?id=407748997003

Llorca, A., Richaud, M., y Malonda, E. (2017). Parenting Styles, Prosocial, and Aggressive Behavior: The Role of Emotions in Offender and Non-offender Adolescents. Frontiers in Psychology, 8, 1246. https://doi.org/10.3389/fpsyg.2017.01246

Mestre, M., Tur, A., Samper, P. \& Nácher, M. (2007). Estilos de crianza en la adolescencia y su relación con el comportamiento prosocial. Revista Latinoamericana de Psicología. http://www.scielo.org.co/scielo.php?pid=S012005342007000200001\&script=sci_abstract\&tlng=es.

Mestre, M., Samper, P., \& Frias, D. (2004). Personalidad y contexto familiar como factores predictores de la disposición prosocial y antisocial de adolescentes. Rev. Latinoam. psicol, 445-457.

Mestre, M., Samper, P., Nácher, M., Tur, A., y Cortés, M. (2006). Psychological Processes and Family Variables as Prosocial Behavior Predictors in a Sample of Spanish Adolescents. Psychological Reports, 98(1), 30-36. https://doi.org/10.2466/pr0.98.1.30-36

Montiel Castillo, V., \& González Pérez, M. (2021). Conducta prosocial en adolescentes con discapacidad intelectual leve. Revista Conrado, 17(79), 1521.

Paez, A., \& Rovella, A. (2019). Vínculo de apego, estilos parentales y empatía en adolescentes. Interdisciplinaria, 36(2), 23-38. https://doi.org/10.16888/interd.2019.36.2.2

Peña, P., Villavicencio, L., Palacios, M., y Mora, J. (2015). Maternidad adolescente y estilo de crianza actual. Acción Pedagógica, 24(1), 62-72.

Pinheiro, C., y Assunção, S. (2020). Estilos parentais e comportamento desviante: 
Papel mediador do consumo de álcool em estudantes universitários. Suma Psicológica, 27(2), 98-106. https://doi.org/10.14349/sumapsi.2020.v27.n2.4

Popp, T., Delgado, M., y Wheeler, L. (2019). Mexican-Origin Parents' Stress and Satisfaction: The Role of Emotional Support. Family Process, 58(1), 146-164. https://doi.org/10.1111/famp.12342

Quezada Fernández, J. (2015). Estilos de crianza en familias nucleares con hijos únicos. [Tesis de Maestría, Universidad de Cuenca]. http://dspace.ucuenca.edu.ec/handle/123456789/22286

Ramírez, M. (2005). Padres y desarrollo de los hijos: Prácticas de crianza. Estudios pedagógicos (Valdivia), 31(2), 167-177. https://doi.org/10.4067/S071807052005000200011

Rico Revelo, D., Encinales de la Hoz. \& Mendoza Becerra, M. (2021). Conducta prosocial, empatía y apoyo emocional en organizaciones de víctimas del conflicto armado. Universidad Católica de Colombia.

Rodríguez, L., Mesurado, B., Oñate, M., Guerra, P. \& Menghi, M. (2017). Adaptación de la Escala de Prosocialidad de Caprara en Adolescentes Argentinos. Revista Evaluar, 17, 177-187. https://doi.org/10.35670/16674545.v17.n2.18730

Valdivia, S., Albo, J., Cruz, A., Villanueva, J., y Jiménez, I. (2020). Psychological Flexibility with Prejudices Increases Empathy and Decreases Distress Among Adolescents: A Spanish Validation of the Acceptance and Action Questionnaire-Stigma. Frontiers in Psychology, 11, 565638. https://doi.org/10.3389/fpsyg.2020.565638

Velásquez Belmar, P., Villouta González, A., y Opazo Vega, D. (2015). Adaptación y Validación del "Parenting Styles and Dimensions Questionnaire" (PSDQ) (Cuestionario de Dimensiones y Estilos de Crianza) de Robinson y cols. (1995), en padres/madres de niños/as que cursan el nivel preescolar y básico en un grupo de establecimientos municipales de la ciudad de Chillán. [Tesis de Pregrado, Universidad del Bío-Bío]. http://repobib.ubiobio.cl/jspui/bitstream/123456789/622/1/Vel\%C3\%A1squez \%20Belmar,\%20.pdf

Villavicencio Aguilar, C., Armijos Piedra, T., y Castro Ponce, M. (2020). Conductas disruptivas infantiles y estilos de crianza. Revista Iberoamericana de Psicología, 13(1), 139-150. https://doi.org/10.33881/2027-1786.rip.13113 
Yoo, H., Feng, X., y Día, R. (2013). Adolescents’ Empathy and Prosocial Behavior in the Family Context: A Longitudinal Study. Journal of Youth and Adolescence, 42(12), 1858-1872. https://doi.org/10.1007/s10964-012-9900-6

\section{Ciencia Digital

El artículo que se publica es de exclusiva responsabilidad de los autores y no necesariamente reflejan el pensamiento de la Revista Conciencia Digital.

\section{Liencia}

El artículo queda en propiedad de la revista y, por tanto, su publicación parcial y/o total en otro medio tiene que ser autorizado por el director de la Revista Conciencia Digital.
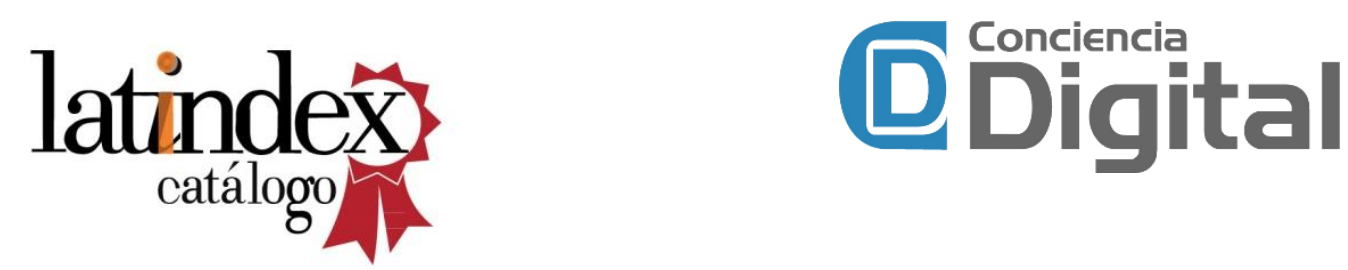

Indexaciones

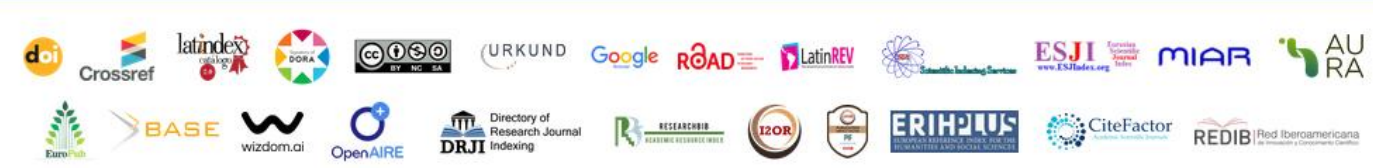

